\title{
Design and Fabrication of a Composite Morphing Radiator Panel Using High Conductivity Fibers
}

\author{
Matthew T. Wescott*, J. Scott McQuien ${ }^{\dagger}$, Christopher L. Bertagne ${ }^{\ddagger}$, John D. Whitcomb ${ }^{\S}$, \\ Darren J. Hartl ${ }^{\mathbb{I}}$ \\ Texas A\&M University, College Station, Texas, 77843 \\ Lisa R. Erickson" \\ NASA Johnson Space Center, Houston, Texas, 77058
}

\begin{abstract}
Upcoming crewed space missions will involve large internal and external heat loads and require advanced thermal control systems to maintain a desired internal environment temperature. Radiators with at least 12:1 turndown ratios (the ratio between the maximum and minimum heat rejection rates) will be needed. However, current technologies are only able to achieve turndown ratios of approximately 3:1. A morphing radiator capable of altering shape could significantly increase turndown capabilities. Shape memory alloys offer qualities that may be well suited for this endeavor; their temperature-dependent phase changes could offer radiators the ability to passively control heat rejection. In 2015, a morphing radiator prototype was constructed and tested in a thermal vacuum environment, where it successfully demonstrated the morphing behavior and variable heat rejection. Newer composite prototypes have since been designed and manufactured using two distinct types of SMA materials. These models underwent temperature cycling tests in a thermal vacuum chamber and a series of fatigue tests to characterize the lifespan of these designs. The focus of this paper is to present the design approach and testing of the morphing composite facesheet. The discussion includes: an overall description of the project background, definition of performance requirements, composite materials selection, use of analytic and numerical design tools, facesheet fabrication, and finally fatigue testing with accompanying results.
\end{abstract}

\section{Introduction}

\section{A. Project Description}

The project focuses on the design, fabrication, and demonstration in a thermal vacuum test of a structurally and thermally optimized radiator facesheet prototype, incorporating the temperature-dependent behavior of shape memory alloys (SMAs). This novel type of radiator is known as a morphing radiator $;{ }^{1}$ it employs SMA materials to passively change the radiator shape to adapt its rate of heat rejection to vehicle requirements. The prototype radiator facesheet has a naturally closed position (similar to a cylinder) in a cold environment. As the radiator's temperature gradually rises, SMAs affixed to the facesheet contract, leading to a change of radiator radius; and increasing the exposure of the radiator inner surface view factor to space and allowing it to reject more heat. SMA materials have been used in a number of aerospace applications and are robust, ${ }^{2,3}$ with an actuation fatigue life exceeding 1E4-1E6 cycles. This technology addresses several of the Evolvable Mars Campaign's (EMC) needs including: (1) variable heat rejection, (2) lightweight and efficient structures and materials, and (3) reliable mechanisms for long-duration missions beyond low earth orbit (LEO). These missions require a thermal control system (TCS) with a maximum to minimum heat rejection ratio (i.e. turn down ratio) of 12:1. ${ }^{4}$ The highest turndown ratio a state-of-the-art TCS can achieve is 3:1 ${ }^{5}$ The 12:1 ratio enables a shift in TCS design from the conventional dual loop to a single loop architecture by decreasing the required temperature range of a TCS and increasing the TCS minimum temperature, enabling single loop use of non-toxic working fluids with higher freezing points ${ }^{6}$ Adopting a single loop TCS over a dual loop TCS will reduce the TCS mass by as much as $25 \%$ while also simplifying vehicle design. ${ }^{4}$

Based on system requirements, candidate SMA and facesheet materials have been evaluated and procured. Down-

*Undergraduate Researcher, Department of Aerospace Engineering, 3141 TAMU

$\dagger$ Graduate Research Assistant, Department of Aerospace Engineering, 3141 TAMU

${ }^{\ddagger}$ NASA Space Technology Research Fellow, Department of Aerospace Engineering, 3141 TAMU, AIAA Student Member

$\S$ Professor, Department of Aerospace Engineering, 3141 TAMU, AIAA Member.

IITEES Research Assistant Professor, Department of Aerospace Engineering, 3141 TAMU, AIAA Member.

"Thermal Engineer, Crew and Thermal Systems Division, 2101 NASA Parkway. 
selection of candidate materials relied on analytical predictions of the SMA behavior and predicted radiator actuation obtained via a thermally coupled model for morphing SMA radiators developed at Texas A\&M. The optimal prototype configuration (e.g. size, interface between SMAs and facesheet) was determined with expert input from each collaborator, the coupled thermo-mechanical model, and a series of bench top tests. Thermal vacuum testing of the prototype radiator was conducted to demonstrate operation in a relevant environment and thus raise the technology's readiness level from a TRL2 to TRL3. This development has provided progress toward overcoming a longstanding NASA technical hurdle of demonstrating a practical means to achieve the necessary 12:1 turndown ratio and does so in a passive manner by the inherent behavior of SMAs.

Figure 1 shows a morphing radiator design which combines the temperature induced transformation phenomena from SMAs with a thermally conductive and linearly elastic biasing structure to create a radiator panel that reconfigures passively in response to changes in temperature. The radiator consists of a circular panel fixed along the panel line of symmetry. The panel is given a high-emissivity coating on the inner (concave) surface, shown with dark shading, and low-emissivity coating on the outer (convex) surface, shown with light shading. This surface treatment adds minimal weight for an increase in TCS performance. Shape memory alloy material (wires, strips, or film) attached to the outermost surface of the panel allows the radiator to morph between various shapes depending on the temperature of the panel. When sufficiently cold, the radiator takes on the circular shape shown in Figure 1a. As the temperature in the radiator increases due to a warmer ambient environment and/or increase in the heat load, the SMA begins to open the radiator to an intermediate configuration, such as that shown in Figure 1b. Figure 1c shows the fully-open, maximum-heat-rejection shape. This morphing behavior is intended to be fully reversible: a subsequent decrease in temperature will cause the radiator to return to the minimum heat rejection shape.

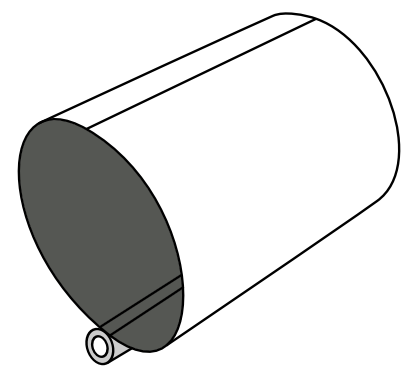

(a) Closed shape for minimum heat rejection.

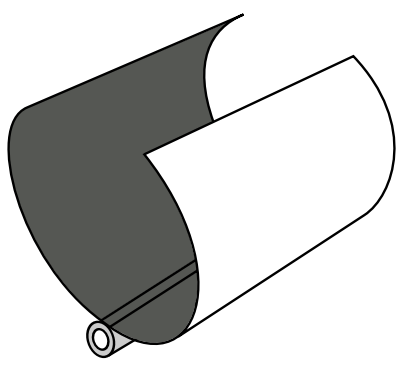

(b) Semi-open shape for intermediate heat rejection.

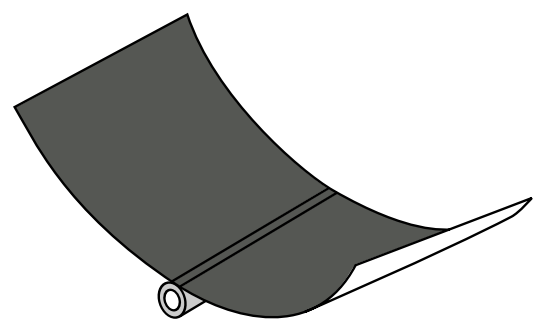

(c) Open shape for maximum heat rejection.

Figure 1: Schematic representation of a flexible morphing radiator panel. Light and dark shading represents low- and high-emissivity coatings, respectively.

\section{B. Technical Approach}

Research began with definition of the facesheet and prototype radiator requirements that account for a space environment and large vibrational loads to be survived during launch. Candidate materials were selected based on their ability to satisfy the design requirements. The facesheet requires three contradictory responses: sufficient stiffness to provide spring force for returning the radiator to its closed cold shape, ample compliance to deform to its open hot shape, and adequate thermal conductivity to transport heat out of the fluid loop. Options included composite and metallic materials, and potentially a combination of the two in a laminate. SMA materials were initially selected from fatigue-resistant commercially available options. These candidate materials were modeled and tested to further evaluate their suitability using collaborator expertise and Texas A\&M's existing coupled FEA/thermally coupled model for general SMA radiators. Validation and further refinement of the FEA/thermal model was performed parallel to this effort using existing NASA Space Technology Research Fellowship funds but are not addressed in this short paper. After material down-selection the FEA/thermal model was employed to find optimal parameters for the prototype radiator's design (e.g. size, conductivity between SMAs and facesheet). The SMA facesheet assembly method was then determined. Novel arrangements of composite fibers and SMA material were explored to provide the necessary combination of actuation, conductivity, stiffness, and strength. Once fabricated, the bench-top test articles were tested in two steps: (1) actuation controlled via electrically heated SMA components and (2) high temperature fluid loop testing demonstrating "passive" SMA actuation. These steps provided confidence on the actuating behavior, and Step 1 also permitted straightforward testing of a high number of cycles, addressing SMA and morphing radiator fatigue concerns. Finally, a mission-inspired 
low temperature test article was fabricated and tested in a thermal vacuum chamber at Johnson Space Center, raising the TRL of the technology from 2 to 3 .

\section{Analysis and Design}

The design space was characterized through a combination of analytical, numerical, and experimental methods. Several design tools were developed in order to predict both the mechanical and thermal properties of a specified facesheet material, geometry, and layup; i.e., the number and orientation of each lamina in the overall laminate. In particular, a straightforward analytical model based on classical Laminated Plate Theory (LPT) was developed to characterize the mechanical behavior of a given facesheet layup, including prediction of elastic response and evaluation of laminate failure criteria. Laminate thermal conductivity and maximum bending curvature are calculated for each specified layup. Laminate thermal conductivity differs from the base material (i.e., fiber and resin matrix) properties as the laminate is made up of a combination of layers at different orientations. Following the development of mechanical design tools, several benchtop experiments were subsequently performed on test specimens to validate the predictions of these tools. In addition, a higher-fidelity numerical model was developed in Abaqus ${ }^{\circledR}$ in order to predict the thermal response (i.e. temperature distribution and heat rejection rate) of a given facesheet design operating in a radiative environment with a known sink temperature. ${ }^{7-9}$ Together, these tools were used to drive the design of three test articles which would be fabricated for fatigue and thermal testing.

\section{A. Mechanical Analysis}

Within the LPT model, maximum bending curvature and effective laminate thermal conductivity here calculated for each specified layup. This is important because laminate thermal conductivity obviously differs from the base material (i.e. lamina) properties as the laminate is made up of a combination of laminae oriented in different directions. Maximum bending curvature is calculated based on first ply failure considering ultimate strain failure criteria in both axial and transverse tension and compression. The LPT model is based on the classical laminated plate equation,

$$
\left\{\begin{array}{l}
N \\
M
\end{array}\right\}=\left[\begin{array}{ll}
A & B \\
B & D
\end{array}\right]\left\{\begin{array}{l}
\varepsilon^{0} \\
\kappa
\end{array}\right\}
$$

which solves for the midplane strains and curvatures, $\varepsilon^{0}$ and $\kappa$, respectively, of a given layup in terms of force and moment stress resultants $N$ and $M$. Layup mechanical properties are calculated and assembled into the so-called $A B D$ matrix. Given the radiator configuration and the distributed bending moment the SMA provides, the applied load is assumed to have only one term, represented by the stress resultant $M_{x}$. All other stress resultants are assumed to be zero. The midplane strains and curvatures are computed in terms of $M_{x}$, and are then used to calculate the global strains in each lamina using the equation,

$$
\left\{\begin{array}{l}
\varepsilon_{x x} \\
\varepsilon_{y y} \\
\varepsilon_{x y}
\end{array}\right\}_{i}^{G}=\left\{\begin{array}{l}
\varepsilon_{x x}^{0} \\
\varepsilon_{y y}^{0} \\
\varepsilon_{x y}^{0}
\end{array}\right\}+z_{i}\left\{\begin{array}{c}
\kappa_{x} \\
\kappa_{y} \\
\kappa_{x y}
\end{array}\right\}
$$

where the subscript $i$ denotes the index of the lamina and $z_{i}$ is the distance of the midplane of lamina $i$ from the midplane of the laminate. These laminae strains are then transformed into the local orientation of the lamina by writing them in tensor form and using the tensor transformation law,

$$
\varepsilon_{i j}^{L}=a_{i k} a_{j l} \varepsilon_{k l}^{G}
$$

where the $a_{i j}$ matrices are simple rotation matrices about the transverse axis, computed using the orientation angle of each lamina. The local laminae coordinate systems are defined by the first axis running parallel to the fibers, with the second axis orthogonal to the first in the plane of the lamina. Once the strains are obtained in the local coordinate system of each lamina, each normal strain is set equal to the corresponding failure strain, in both tension and compression. The four resulting equations are evaluated to find the $M_{x}$ required to fail the lamina in all modes. This is done for each lamina, and the first ply failure is found by the smallest required $M_{x}$. This $M_{x}$ is then re-substituted into Equation 1 to yield midplane curvature $\kappa_{x}$ at which the given laminate will fail. This $\kappa_{x}$ describes, through simple geometric calculations, the maximum angle to which the given laminate can open from the reference cylindrical shape.

The given facesheet's effective thermal conductivity per unit width (denoted by $K t$ ) in the circumferential direction is evaluated by transforming each lamina thermal conductivity into global coordinates following reverse procedure as in Equation 3, multiplying by the lamina thickness (t), and then summing over each lamina:

$$
K t=\sum_{i=1}^{N} k_{i} t_{i}
$$


From these operations, the failure stress $M_{x}$, corresponding midplane curvature $K_{x}$ and thermal conductivity (Kt) for a given laminate are computed.

\section{B. Composite Material Selection}

Certain classes of carbon fiber reinforced composites (i.e., those made from pitch based fibers) are suitable for this application as they are capable of extremely high thermal conductivities (TC), high stiffness, and are lightweight. Simply manufacturing the facesheet out of the correct composite material can satisfy all of the requirements. In addition, this type of composite can be produced in a unidirectional tape manner allowing it to be configured via appropriate or optimized layups to tailor resulting thermal and structural properties. Considering the facesheet as a layup of carefully oriented plies of unidirectional fibers, the required stiffness and TC can be achieved.

The material selected is a pre-impregnated unidirectional composite tape made up of RS-3C ${ }^{\circledR}$ resin (produced by TenCate Advanced Composites), and K13D2 $\mathrm{U}^{\circledR}$ pitch carbon fibers (produced by Mitsubishi Rayon). The prepreg is assembled and prepared by TenCate Advanced Composites ${ }^{\circledR}$. The material system has previously been proven in the space environment, and the resin meets outgassing requirements for space flight. It is also capable of being processed using out-of-autoclave curing cycles under vacuum pressure. Key material properties include lamina thermal conductivity of $480 \mathrm{~W} / \mathrm{mK}$, and stiffness of $465 \mathrm{GPa}$ at $0^{\circ}$ (fiber direction).

The design tools were validated through experimental fabrication of test facesheet panels. Experimental results further narrowed down the number of possible layup combinations. In particular, it was shown that layups of only $0^{\circ}$ oriented laminae lacked sufficient transverse in plane durability and were highly susceptible to handling-induced cracking. The optimum layup was found to consist of some number of $0^{\circ}$ lamina sandwiched in between non $0^{\circ}$ lamina. Further experimental testing showed that using only laminae outside othe the $0^{\circ}$ laminae causes matrix cracking within the $90^{\circ}$ plies. In addition, since the thermal conductivity of the constituent resin is much lower than that of the fibers, the $90^{\circ}$ laminae are almost completely thermally negligible. Lastly, the non $0^{\circ}$ laminae must not lead to significant thermal stresses and/or deformation coupling with bending. With these considerations in mind, experimental results have led to the selection of layups that incorporate two to three $0^{\circ}$ laminae (oriented in the circumferential direction) in between layers of $\pm 45^{\circ}$ laminae in an anti-symmetric fashion. Key material properties are presented in Tables 1 and $2 .{ }^{10}$

Table 1: Fiber properties of various high-conductivity pitch fibers produced by Mitsubishi Rayon ${ }^{\circledR}$.

$\begin{array}{cccc}\begin{array}{c}\text { Fiber } \\ K 13 D 2 U^{\circledR}(2 \mathrm{~K})\end{array} & \begin{array}{c}\text { Modulus } \\ 930 \mathrm{GPa}\end{array} & \begin{array}{c}\text { Thermal Conductivity } \\ \text { Approx. Fiber Cost }\end{array} \\ \mathrm{K} 13 \mathrm{C} 2 \mathrm{U}^{\circledR}(2 \mathrm{~K}) & 900 \mathrm{GPa} & 620 \mathrm{~W} / \mathrm{m} \cdot \mathrm{K} \cdot \mathrm{K} & \$ 1,000 / \mathrm{lb} \\ \mathrm{K} 13916^{\circledR}(16 \mathrm{~K}) & 760 \mathrm{GPa} & 200 \mathrm{~W} / \mathrm{m} \cdot \mathrm{K} & \$ 800 / \mathrm{lb} \\ & & \$ 75 / \mathrm{lb}\end{array}$

Table 2: Effective laminate properties in the fiber direction.

$\begin{array}{cccc}\text { Material } & \text { Modulus } & \text { Thermal Conductivity } & \text { Ultimate Strain } \\ \text { K13D2U Laminate } & 590 \mathrm{GPa} & 478 \mathrm{~W} / \mathrm{m} \cdot \mathrm{K} & 0.4 \% \\ \text { K13C2U Laminate } & 550 \mathrm{GPa} & 367 \mathrm{~W} / \mathrm{m} \cdot \mathrm{K} & 0.4 \%\end{array}$

\section{Thermal Analysis}

A high-fidelity thermal model was developed in $\mathrm{Abaqus}^{\circledR}$ to predict the steady-state thermal response of a given facesheet design.$^{7-9}$ This model was then used to investigate maximum and minimum heat rejection of a given layup. Maximum bending curvature is used in conjunction with a specified radiator diameter to determine the maximum heat rejection of the given facesheet (open configuration). A two-dimensional thermal analysis is performed on a representative geometry using the calculated thermal conductivity properties and including radiation boundary conditions. Considering heat rejection in the closed configuration in addition to the open configuration, turndown ratio can be determined.

Figure 2 shows the results of a number of individual thermal analyses for a small selection of important layups. Each specific layup is analyzed at an open configuration unique to it; some panels, based on their layup, may be able to open further than others. In addition, each layup has a unique effective thermal conductivity. In Figure 2, each point represents a different radiator diameter, increasing from upper left to lower right in eighth inch increments from 1 to 4 inches. As radiator diameter increases, the specific layup is able to open further, and heat rejection is increased. However, as the facesheet panel becomes larger, the fin efficiency decreases significantly. 


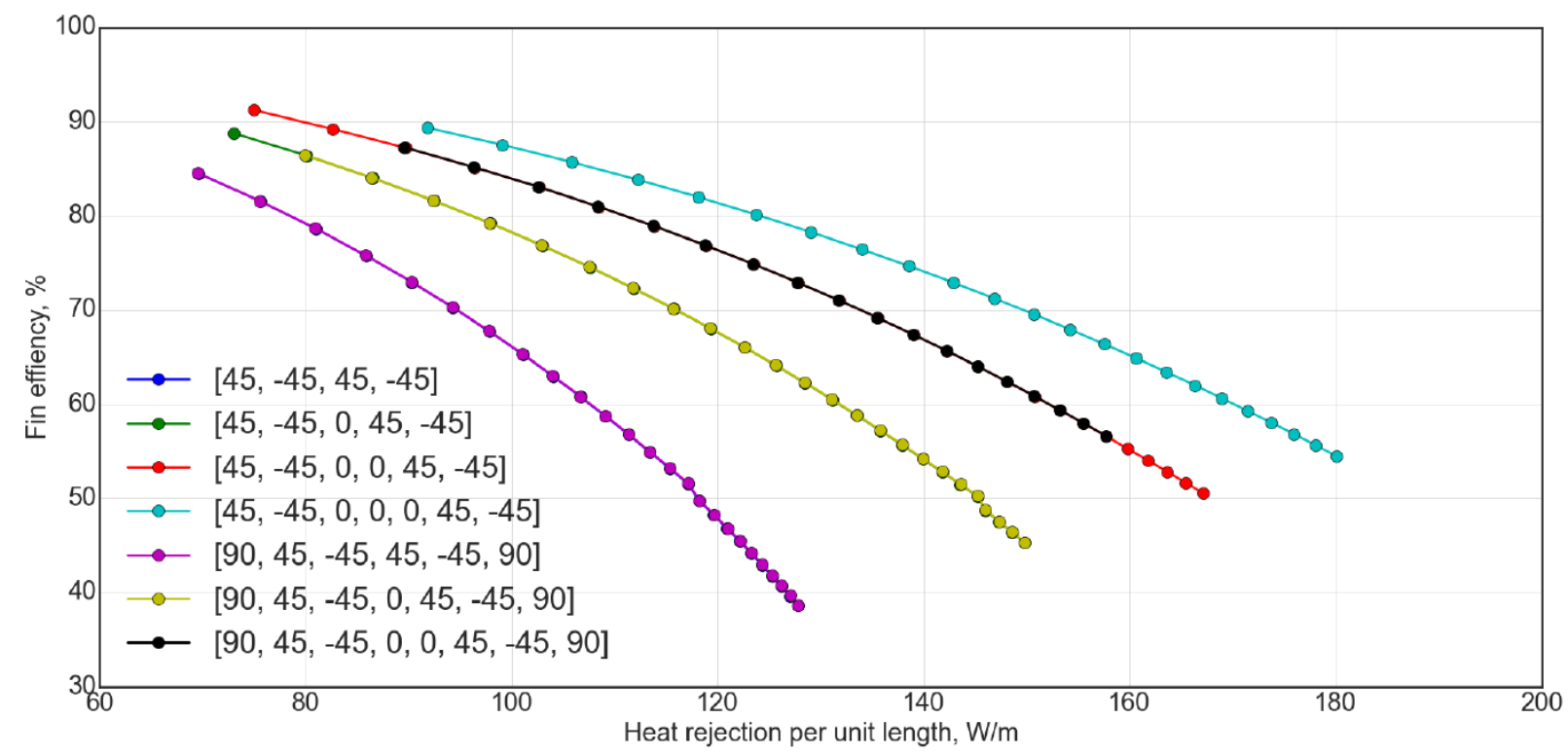

Figure 2: Maximum heat rejection plotted versus fin efficiency for specified layups formed into cylindrical reference shapes of increasing diameter.

\section{Panel Fabrication}

As stated previously, the chosen material system allows for vacuum-assisted out-of-autoclave curing. This permits the use of conventional vacuum bagging techniques and vacuum equipped oven. Fabrication of the facesheet largely followed the standard out-of-autoclave composite fabrication methodology. For the facesheet to provide the correct biasing force for SMA actuation, the composite facesheet was fabricated such that its stress free (cured) state is its closed cylindrical configuration. Accordingly, a fabrication tool known as a mandrel was designed to allow for the composite to be laid up, vacuum bagged, and cured in the correct configuration. Since the facesheet is not fully cylindrical (i.e., a small gap exists between open ends), the material is able to slide against the tool during curing to avoid thermal hoop stresses caused by the mandrel's thermal expansion. Figure 3 shows a facesheet in its curing configuration before being enclosed in the breather fabric and vacuum bag.

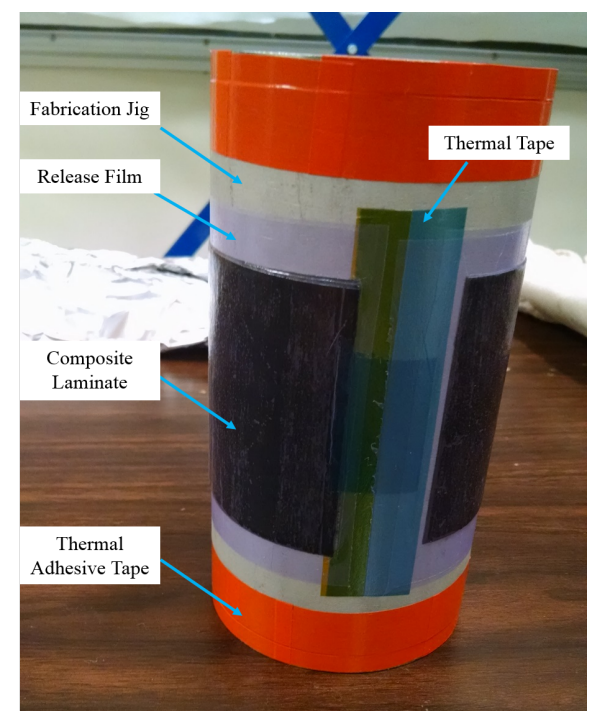

Figure 3: Sample panel after laying up on the mandrel and before being vacuum bagged.

The material layup process in the closed, circular configuration, and custom fabrication jig with modified vacuum bagging procedure is detailed in Figure 4. Figure 5 show the completed vacuum bagging process and attached vacuum 
port. Then entire assembly is then placed in a large industrial oven for curing.

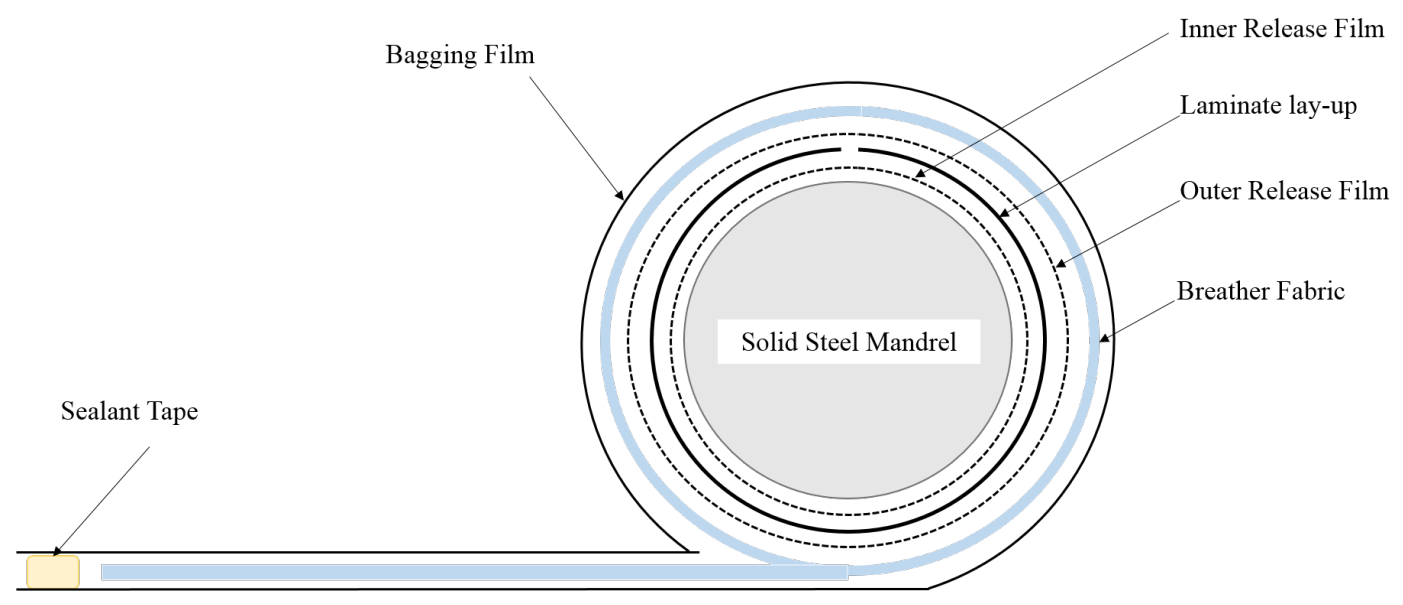

Figure 4: Cross-section of the mandrel, laminate, vacuum bag, and other fabrication necessities.

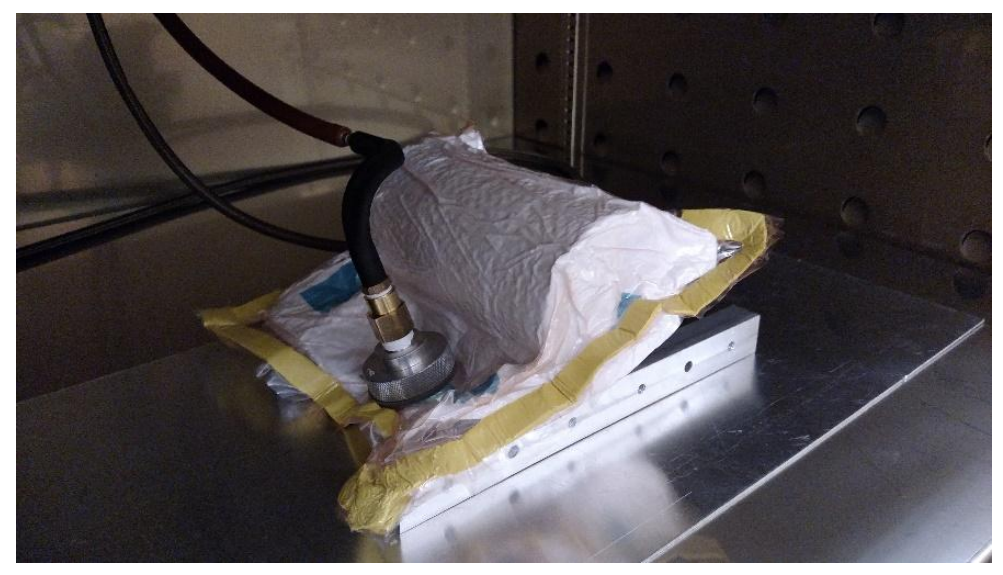

Figure 5: Panel during fabrication, after applying the vacuum and placing in the curing oven.

After curing and cooling the assembly is removed and the finished composite panel is extracted from the mandrel. Figure 6 shows three finished composite specimens of different ply layups. The layup originally proposed for fabrication was a $[ \pm 45 / 0 / 0 / 0 / \pm 45]$ stacking sequence. However, initial bending of test specimens revealed fiber micro-buckling on the outer facesheet. Thus, 90 degree plies were added to the stacking sequence to prevent micro-buckling while adding no significant increase to the bending stiffness of the overall panel.

\section{A. Test Articles}

A total of three different test articles were fabricated for the thermal vacuum chamber tests using the procedure described in Sec. III. Table 3 gives an overview of each of the test articles. The first row gives the layup of the unidirectional fiber-reinforced composite used for each test article. Angles are defined with respect to the circumferential direction (i.e. the fibers of a $0^{\circ}$ ply are along the circumference of the panel and the fibers of a $90^{\circ}$ ply are oriented parallel to the flow tube). The key mechanical and thermal properties of the panel, namely, bending stiffness and effective conduction coefficient per unit length (denoted by $K t$ ), are also listed for each panel, along with the primary dimensions. The first two test articles, A and B, were nearly identical apart from the layup: Test Article A used a layup with a single ply oriented in the circumferential direction (i.e., $0^{\circ}$ ); while the layup for Test Article B included an additional circumferential ply. 


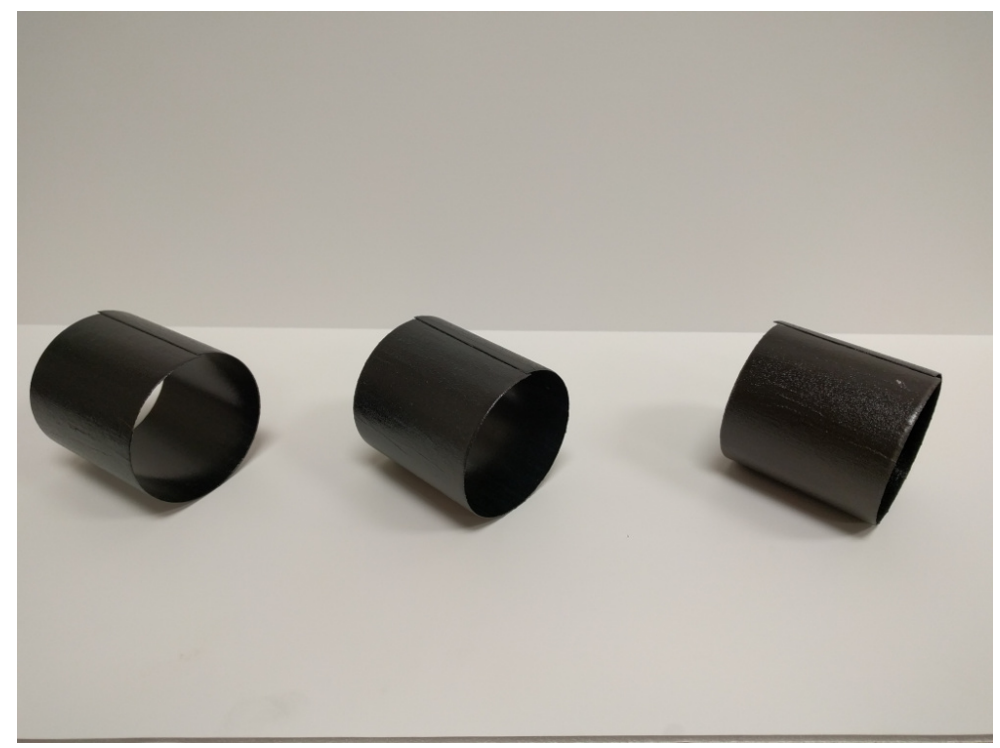

Figure 6: Completed panels. Left to right: $[90 / \pm 45 / 0 / \pm 45 / 90]^{\circ},[90 / \pm 45 / 0 / 0 / \pm 45 / 90]^{\circ},[90 / \pm 45 / 0 / 0 / 0 / \pm 45 / 90]^{\circ}$.

Table 3: Overview of the three test articles fabricated for the thermal vacuum chamber tests.

\begin{tabular}{rlll}
\hline & Test Article A & Test Article B & Test Article C \\
\hline Layup & {$[90 / \pm 45 / 0 / \pm 45 / 90]$} & {$[90 / \pm 45 / 0 / 0 / \pm 45 / 90]$} & {$[90 / \pm 45 / 0 / 0 / 0 / \pm 45 / 90]$} \\
Bending stiffness & $0.44 \mathrm{~Pa} \cdot \mathrm{m}^{3}$ & $0.82 \mathrm{~Pa} \cdot \mathrm{m}^{3}$ & $1.42 \mathrm{~Pa} \cdot \mathrm{m}^{3}$ \\
Cond. coeff. per unit width $(K t)$ & $0.091 \mathrm{~W} / \mathrm{K}$ & $0.121 \mathrm{~W} / \mathrm{K}$ & $0.152 \mathrm{~W} / \mathrm{K}$ \\
Closed diameter & $3.0 \mathrm{in}$ & $3.0 \mathrm{in}$ & $3.5 \mathrm{in}$ \\
Width & $3.0 \mathrm{in}$ & $3.0 \mathrm{in}$ & $4.0 \mathrm{in}$ \\
SMA type \& qty. & Wires $(x 18)$ & Wires $(x 36)$ & Strips $(\mathrm{x} 8)$ \\
SMA phase at room temp. & Austenite & Austenite & Martensite \\
\hline
\end{tabular}

\section{Fatigue Tests}

\section{A. Test Setup}

A primary concern for the design of a composite morphing radiator is the fatigue life of the composite facesheet. A target minimum of 370 cycles was provided as a requirement by NASA JSC, taken from the lightweight radiator requirements from development in 2004-2005. ${ }^{11}$ A benchtop fatigue test stand was constructed consisting of low friction pulleys, roller bars, and in-line Shimpo force gauge ${ }^{\circledR}$. Using this setup, displacements similar (but not identical) to those observed during radiator actuation could be applied in a fully controlled and repeatable manner. As is shown in Figure 7, the composite specimen is mounted using risers and ultra high molecular weight polyethylene Dyneema ${ }^{\circledR}$ string is then attached under the terminal blocks and connected through the pulley system to the bottom of the platform where the force gauge is connected.

As seen in Figure 8, a turnbuckle is attached in line with the pulley system. As the turnbuckle is adjusted (turned), incremental measurements of displacement and force were recorded, corresponding to "characterization" of the panel stiffness. For the fatigue cycling portion of the experiment, the turnbuckle is opened completely and the force gauge is repeatedly moved up to and away from the hard-stop plate. This process is repeated for the desired number of cycles. The incremental force and displacement portion is then repeated followed by another set of cycles. This is described in more detail below. 


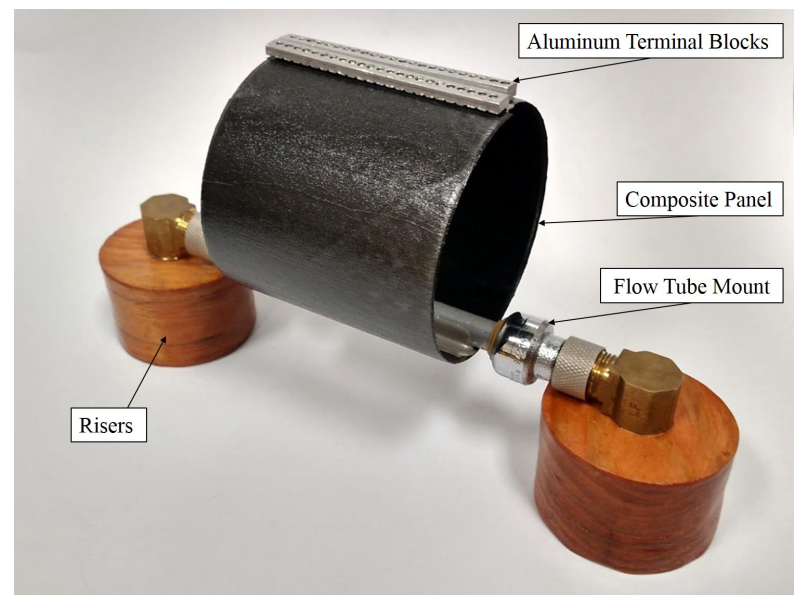

(a)

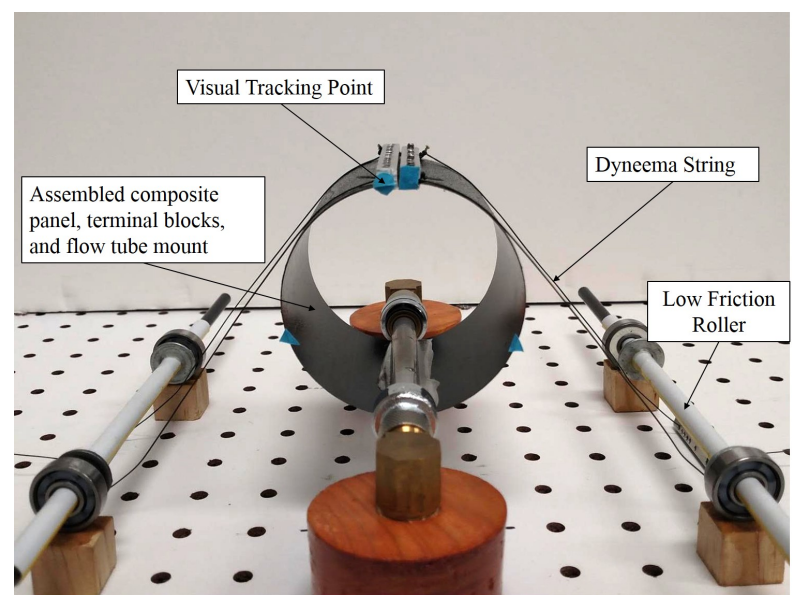

(b)

Figure 7: Photographs of fatigue test article.

As mentioned, the loading applied during the fatigue testing using the Dyneema strings attached to the ends of the panels is not identical to that applied by the SMA wires/strips during morphing radiator actuation. The former corresponds more closely to nonlinear tip loaded bending of a cantilevered beam, while the latter corresponds to nearly constant moment loading throughout the panel. However, it is essential to characterize the fatigue response of the panel under at least local loading similar to that seen during actuation. To determine the appropriate string-loaded deflection magnitude corresponding to the same maximum strains generated during SMA actuation, an Abaqus model was developed. The results of this numerical design analysis are shown in Figure 9. Though shown here qualitatively using a contour image, the string-driven panel edge displacements for strain matching at the fixed root were determined quantitatively to within $0.01 \mathrm{~mm}$. Note that strains are shown as resolved into a fixed global coordinate system and therefore only the values of strains at the panel line of symmetry (i.e., the point of highest strain being sought) are meaningful.

\section{B. Results \& Discussion}

With regard to demonstrating structural robustness under cyclic fatigue loading up to 370 full actuation cycles, the primary focus was on Test Article B previously discussed. This panel included two circumferentially aligned $\left(0^{\circ}\right)$ laminae near the middle of the layup, and was thus stiffer (and more likely to experience damage and failure) than Test Article A, which had only a single circumferentially aligned ply. The specific cyclic loading history ap-

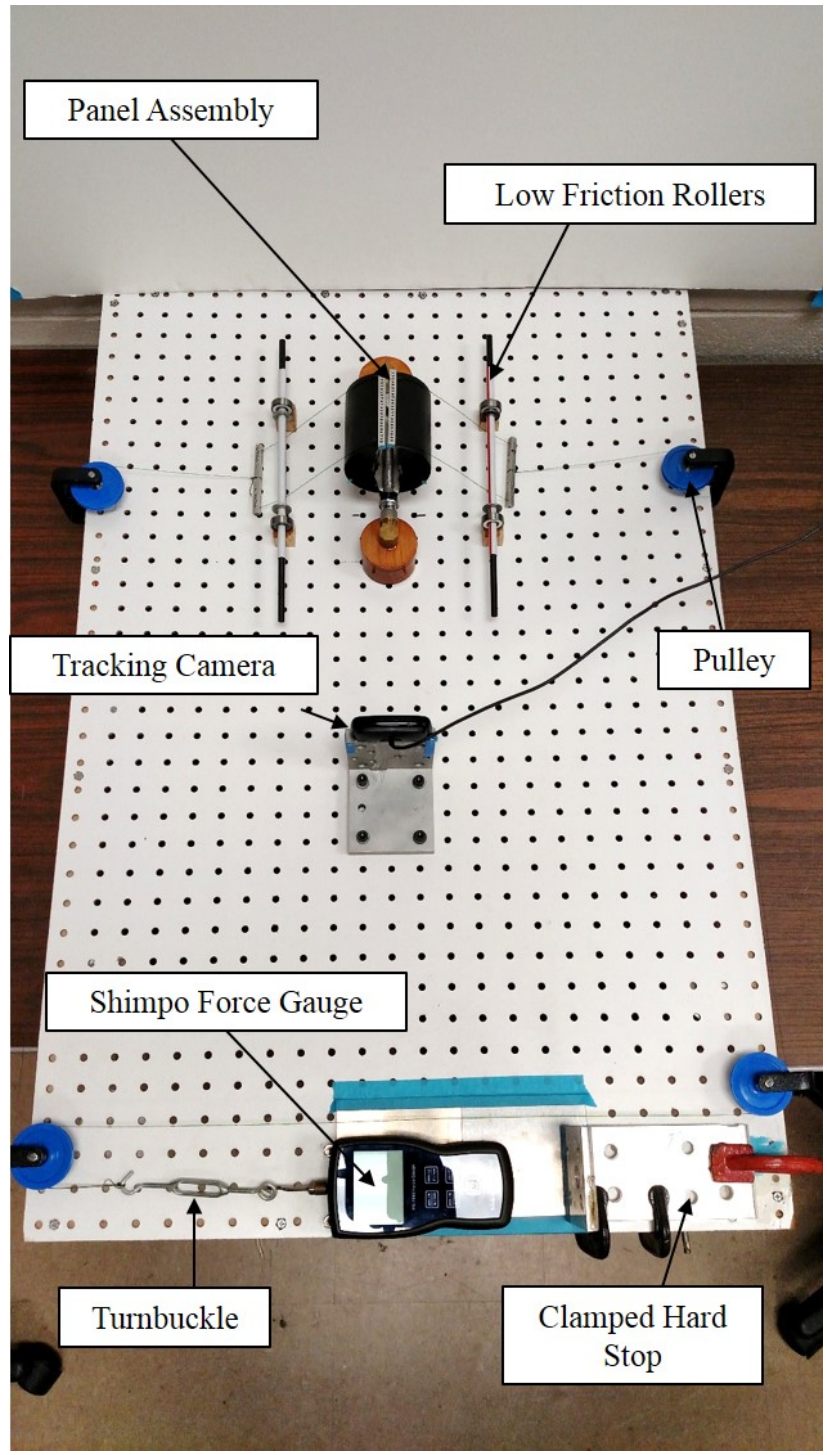

Figure 8: Overview of fatigue test setup. 


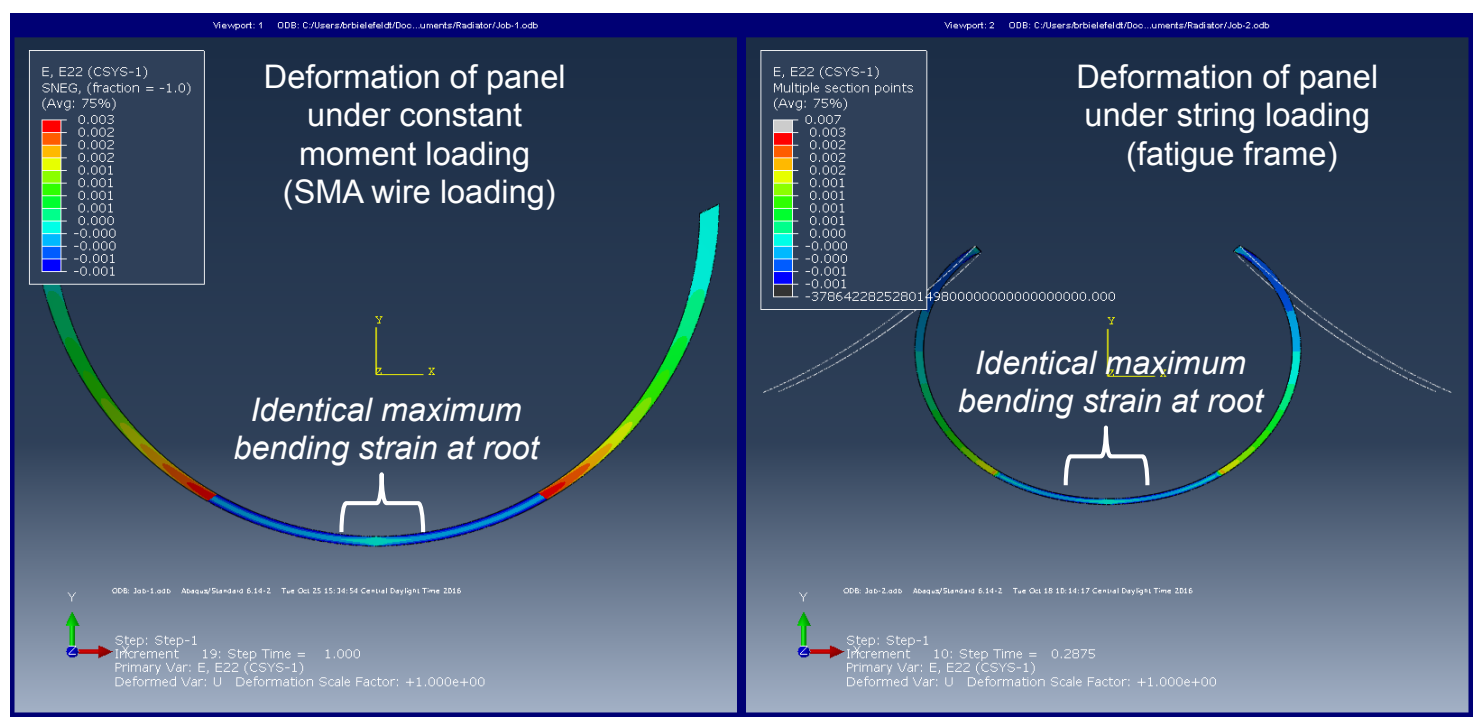

Figure 9: Results of FEA for determining the deflection on the fatigue frame that matched the same maximum panel strain as applied during SMA actuation in the thermal vacuum chamber. Strains are resolved into a fixed global coordinate system; only the strains fields near the line of symmetry (the point of interest) are quantitatively meaningful.

plied is schematically illustrated in Figure 10. Prior to any deformation after fabrication (i.e., with the panel in its virgin state), an initial characterization load/unload cycle was applied to an intermediate deflection level using the experimental fatigue apparatus. This provided a baseline stiffness response and also initialized the test setup (tightened the Dyneema strings, etc.). The loading portion of this initial cycle is shown as the $N=0$ curve in Figure 11a. For this test, the loading characterization was performed by applying a $\sim 50 \mathrm{~mm}$ deflection via the turnbuckle.

Full actuation cyclic loading is then applied for $\Delta N$ cycles, during which force-deflection data was not rigorously logged. The full actuation deflection was $78.4 \mathrm{~mm}$, as determined from the Abaqus analysis of Figure 9. After this cycling, an additional characterization cycle consisting of intermediate deflection levels is performed once more, the force-deflection data logged, and the stiffness assessed. The process was repeated up to $370 \mathrm{cycles}$, and then eventually to 400 cycles, with $\Delta N$ generally increasing from $N=0$ to $N=400$.

The key results from the stiffer Test Article B are shown in Figure 11. Most importantly, the panel is able to survive full radiator actuation strains for up to 400 cycles of loading, which was critical to demonstrating the feasibility of the concept. However, some evolution during cycling loading is clearly observed, with the strongest effects observed in the first five cycles and some continuing evolution up to 100 cycles. This can be seen in both the shift in the reference displacement of the panel in Figure 11a and in the change of stiffness in Figure 11b. The latter is indicative of some damage being generated in the panel. Given the loading method of the fatigue apparatus, this corresponds to damage being formed near the flow tube or root of the panel. Note that stiffness was calculated in the range of 5-10 $\mathrm{N}$ of loading to avoid the overall

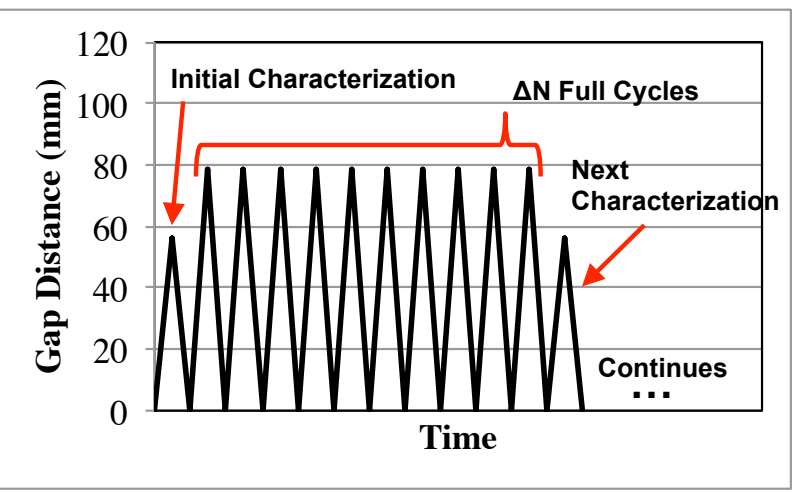

Figure 10: Schematic description of loading applied Test Article B. Data is logged and plotted for "characterization" cycles but not for "full" cycles. nonlinearity observed when examining the entire loading curve. After 100 cycles, the panel appears to have reached a steady-state response where the stiffness is reduced to $\sim 72 \%$ of its original value, but catastrophic failure is never observed. 
Given the success of the Test Article B configuration under cyclic loading, it is of interest to also consider the cycling loading of the more compliant Test Article A configuration (i.e., a single circumferentially aligned ply) cycled to even higher deflections. Since the stiffness of Test Article A is approximately half that of Test Article B, for this case the fatigue loading deformation was approximately doubled to $160 \mathrm{~mm}$. Characterization deformation was also increased to $\sim 80 \mathrm{~mm}$. Again, the panel survived fatigue loading without catastrophic failure for up to 400 cycles. The results are shown in Figure 12. Note here that full shakedown of the response seems to be completed by 20-50 cycles, where the total panel stiffness has been reduced to $\sim 65 \%$ of its original value. Here stiffness was measured in the loading range of $2-4 \mathrm{~N}$ to avoid nonlinearity effects.

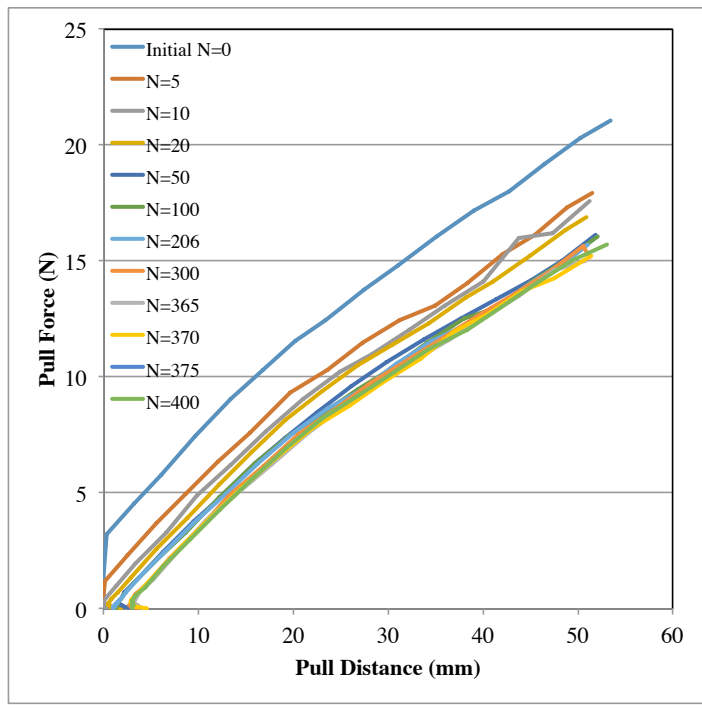

(a) Force-deflection curves as loading cycles increase

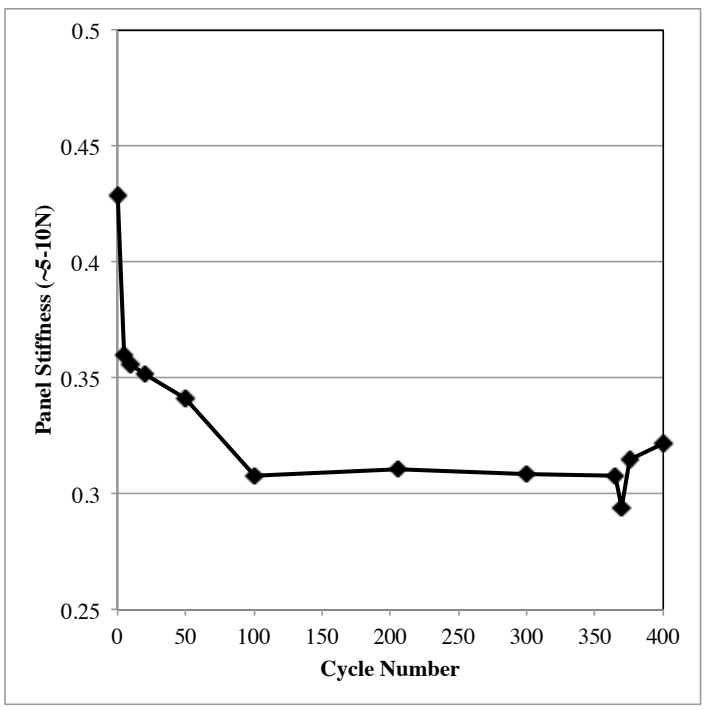

(b) Change in stiffness as loading cycles increase

Figure 11: Fatigue testing results for morphing radiator panel with two circumferentially aligned plies (Test Article B).

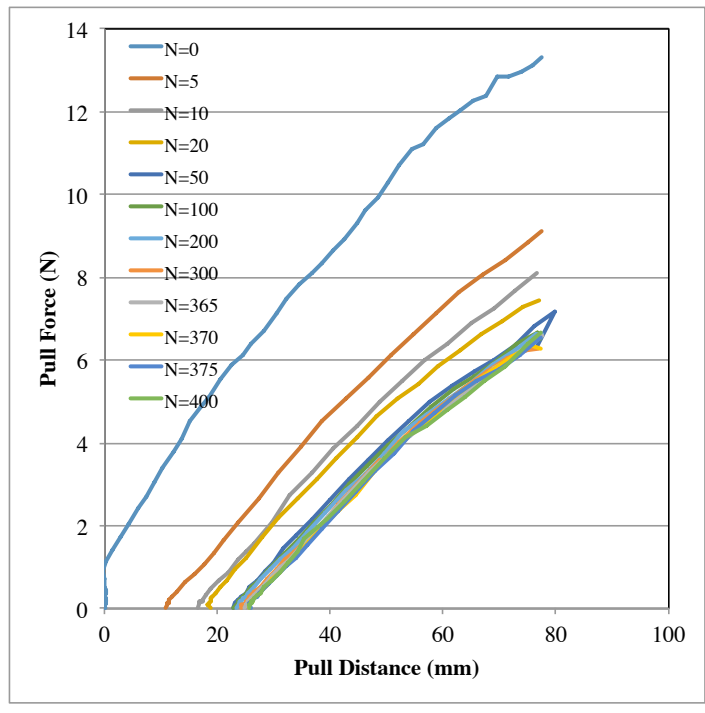

(a) Force-deflection curves as loading cycles increase

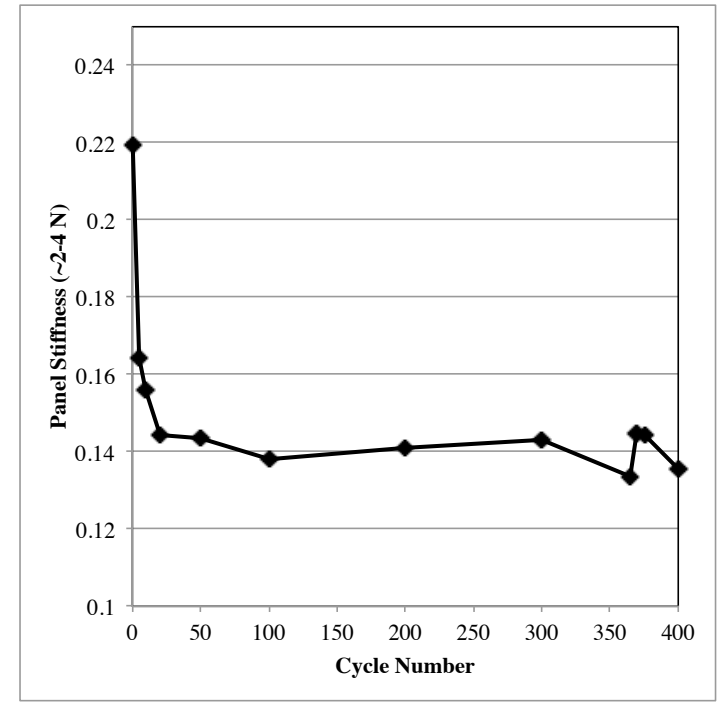

(b) Change in stiffness as loading cycles increase

Figure 12: Fatigue testing results for morphing radiator panel with one circumferentially aligned ply (Test Article A) loaded to $\sim$ double the maximum root strain as in thermal vacuum chamber testing. ${ }^{12}$ 


\section{Summary \& Future Work}

A morphing radiator has been developed in response to the clear need of a next generation TCS capable of higher turndown ratios. As was demonstrated in 2015 through thermal vacuum chamber testing, further refinement of the facesheet material selection could potentially achieve the goal of a 12:1 turndown ratio ${ }^{4,13}$ Following careful selection of commercially available high conductivity composite fibers, application of laminated plate theory derived the effective laminate mechanical and thermal conductivity properties. Numerical simulation was used to predict steady-state thermal response of a given composite layup and cylindrical diameter of the facesheet, giving the maximum and minimum heat rejection rates of the open and closed configurations respectively. Combination of these design tools drove the design studies to arrive at an optimal layup and facesheet diameter.$^{7-9}$ Test articles were fabricated using a custom cylindrical mandrel and out-of-autoclave vacuum bagging procedure. Following thermal vacuum chamber testing of these specimens in 2016, the fatigue life of the composite facesheet was determined. ${ }^{12}$ Through numerical simulation of the actuation undergone in thermal testing, the open displacements for strain matching at the fixed root were determined. By then mechanically cycling fresh specimens in tip loaded bending, the equivalent strains were induced. The composite morphing radiator prototypes clearly exhibited the desired range of actuation and minimum 370 cycle fatigue life without failure. The robustness of the composite panels containing two circumferentially aligned $\left(0^{\circ}\right)$ laminae was demonstrated by considering cycling loading up to full actuation strains (at the root) for 400 cycles. Further testing of the more compliant panel containing one circumferentially aligned $\left(0^{\circ}\right)$ ply showed that 400 cycles of twice the actuation defection/strain is also possible. Overall stiffness degradation was 30-40\% across the two panels and exceeded the 370 cycle minimum fatigue life.

Regarding future work, methods whereby the SMA material is more fully integrated into the highly conductive composite laminate will be investigated. This is expected to greatly improve heat transfer between active and passive structural elements, improving performance, while also reducing part count and decreasing complexity of the assembly. Bonding options and, in particular, the challenges of co-curing thermally activating SMA material with a thermally cured composite system will be addressed; options such as post-cure shape set and room temperature cure options will be investigated. In a similar manner, integration of the fluid flow tube and the composite laminate to further improve heat transfer characteristics will be explored.

\section{Acknowledgments}

This work was supported by a NASA Space Technology Research Fellowship (NSTRF) under grant NNX14AM44H. Special thanks to the following: Dr. Mohammad Naraghi and Dr. Ibrahim Karaman (TAMU) for use of their lab facilities and oven. Brent Bielefeldt for his assistance in Abaqus simulations. Jaime Rendon and Pedro Leal for their assistance in the revision process. James Brown, Tobin Barnes, and Peter Grenfell (NASA JSC, Innovation Design Center) for fabricating the terminal blocks for the radiator prototypes. Finite element analysis was performed using a research license for Abaqus granted by Simulia. Code for the simulation coupling framework was written in the Python programming language.

\section{References}

${ }^{1}$ Cognata, T., Hartl, D., Sheth, R., and Dinsmore, C., "A Morphing Radiator for High-Turndown Thermal Control of Crewed Space Exploration Vehicles," Proceedings of 23rd AIAA/AHS Adaptive Structures Conference, Kissimmee, FL, 2015.

${ }^{2}$ Hartl, D. J. and Lagoudas, D., “Aerospace Applications of Shape Memory Alloys," Proceedings of the Institution of Mechanical Engineers, Part G: Journal of Aerospace Engineering, Vol. 221 (Special Issue), 2007, pp. 535-552.

${ }^{3}$ Jani, J. M., Leary, M., Subic, A., and Gibson, M. A., "A review of shape memory alloy research, applications and opportunities," Materials \& Design, Vol. 56, 2014, pp. 1078-1113.

${ }^{4}$ Ochoa, D. A., Vonau, W., and Ewert, M. K., "A Comparison between One- and Two-Loop ATCS Architectures Proposed for CEV," Tech. rep., SAE Technical Paper, 2009.

${ }^{5}$ NASA, “TA 14: Thermal Management Systems,” NASA Technology Roadmaps, July 2015.

${ }^{6}$ Cognata, T. J., Leimkuehler, T., Balasubramaniam, R., Nayagam, V., Hasan, M., and Stephan, R., "A Review of the Experimental and Modeling Development of a Water Phase Change Heat Exchanger for Future Exploration Support Vehicles," 41st Int'l Conf. on Environmental Systems, 2011, pp. 17-21.

${ }^{7}$ Dassault Systèmes of America Corp., Woodlands Hills, CA, Abaqus Analysis User's Manual, 2007.

${ }^{8}$ Bertagne, C., Hartl, D., and Cognata, T., "Analysis of Highly Coupled Thermal-Structural Responses in Morphing Radiative Bodies," Proceedings of 23rd AIAA/AHS Adaptive Structures Conference, Kissimmee, FL, 5-9 January 2015.

${ }^{9}$ Bertagne, C., Sheth, R., Hartl, D., and Whitcomb, J. D., "Simulating Coupled Thermal-Mechanical Interactions in Morphing Radiators," Proceedings of SPIE, Active and Passive Smart Structures and Integrated Systems, Vol. 9431, San Diego, CA, 9-12 March 2015, pp. 94312F-94312F-10. 
10 “Mitsubishi Rayon - Dialead Data Sheet," Tech. rep., Mitsubishi Rayon, 2015.

${ }^{11}$ Tuan, G. C., Westheimer, D. T., Birur, G. C., Beach, D. E., Jaworske, D. A., Peters, W. C., and Triolo, J. J., "Evaluation of Coatings and Materials for Future Radiators,” Tech. rep., SAE Technical Paper, 2006.

${ }^{12}$ Bertagne, C., Chong, J., Whitcomb, J., Hartl, D., and Erickson, L., "Experimental Characterization of a Composite Morphing Radiator Prototype in a Relevant Thermal Environment," Proceedings of 25th AIAA/AHS Adaptive Structures Conference, Grapevine, TX, 9-13 January 2017.

${ }^{13}$ Bertagne, C. L., Chong, J. B., Whitcomb, J. D., Hartl, D. J., Erickson, L. R., and Sheth, R. B., "Experimental Characterization of a Shape Memory Alloy-Based Morphing Radiator," Proceedings of 24th AIAA/AHS Adaptive Structures Conference, San Diego, CA, 4-8 January 2016, p. 1568. 